\title{
SOME $\mathbb{Z}_{n-2}$ TERRACES FROM $\mathbb{Z}_{n}$ POWER-SEQUENCES, $n$ BEING AN ODD PRIME POWER
}

\author{
IAN ANDERSON \\ Department of Mathematics, University of Glasgow, University Gardens, Glasgow G12 8QW, UK \\ e-mail:ia@maths.gla.ac.uk \\ and D. A. PREECE \\ Queen Mary University of London, School of Mathematical Sciences, Mile End Road, \\ London E1 4NS, UK \\ e-mail:D.A.Preece@qmul.ac.uk \\ and \\ Institute of Mathematics, Statistics and Actuarial Science, Cornwallis Building, \\ University of Kent, Canterbury, Kent CT2 7NF, UK
}

(Received 5 November 2008; accepted 17 April 2009)

\begin{abstract}
A terrace for $\mathbb{Z}_{m}$ is an arrangement $\left(a_{1}, a_{2}, \ldots, a_{m}\right)$ of the $m$ elements of $\mathbb{Z}_{m}$ such that the sets of differences $a_{i+1}-a_{i}$ and $a_{i}-a_{i+1}(i=1,2, \ldots, m-1)$ between them contain each element of $\mathbb{Z}_{m} \backslash\{0\}$ exactly twice. For $m$ odd, many procedures are available for constructing power-sequence terraces for $\mathbb{Z}_{m}$; each such terrace may be partitioned into segments, one of which contains merely the zero element of $\mathbb{Z}_{m}$, whereas each other segment is either (a) a sequence of successive powers of an element of $\mathbb{Z}_{m}$ or (b) such a sequence multiplied throughout by a constant. We now adapt this idea by using power-sequences in $\mathbb{Z}_{n}$, where $n$ is an odd prime power, to obtain terraces for $\mathbb{Z}_{m}$, where $m=n-2$. We write each element from $\mathbb{Z}_{n}$ so that they lie in the interval $[0, n-1]$ and then delete 0 and $n-1$ so that they leave $n-2$ elements that may be interpreted as the elements of $\mathbb{Z}_{n-2}$. A segment of one of the new terraces may be of type (a) or (b), incorporating successive powers of 2, with each entry evaluated modulo $n$. Our constructions provide $\mathbb{Z}_{n-2}$ terraces for all odd primes $n$ satisfying $0<n<1,000$ except for $n=127,241,257,337,431,601,631,673,683,911,937$ and 953. We also provide $\mathbb{Z}_{n-2}$ terraces for $n=3^{r}(r>1)$ and for some values $n=p^{2}$, where $p$ is prime.
\end{abstract}

2000 Mathematics Subject Classification. Primary 11A07, secondary 05B30.

1. Basic definitions and notation. Let $\mathbf{a}=\left(a_{1}, a_{2}, \ldots, a_{m}\right)$ be an arrangement of the elements of $\mathbb{Z}_{m}$, and let $\mathbf{b}=\left(b_{1}, b_{2}, \ldots, b_{m-1}\right)$ be the ordered sequence $b_{i}=a_{i+1}-a_{i}$ for $i=1,2, \ldots, m-1$. The arrangement $\mathbf{a}$ is a terrace for $\mathbb{Z}_{m}$, with $\mathbf{b}$ as the corresponding 2-sequencing or quasi-sequencing for $\mathbb{Z}_{m}$, if the sequences $\mathbf{b}$ and $-\mathbf{b}$ between them contain exactly two occurrences of each element $x$ from $\mathbb{Z}_{m} \backslash\{0\}$. A $\mathbb{Z}_{m}$ terrace is directed $[\mathbf{8}]$ if all the elements in its 2 -sequencing $\left(b_{1}, b_{2}, \ldots, b_{m-1}\right)$ are distinct. For $m$ odd, a $\mathbb{Z}_{m}$ terrace is

- anti-directed if its 2-sequencing contains only $(m-1) / 2$ distinct elements; i.e. if the element $x$ appears in the 2-sequencing it does so twice, whereas $-x$ appears not at all; 
- half-and-half [1] if for each element $x$ of $\mathbb{Z}_{m} \backslash\{0\}$, the set $\left\{b_{1}, b_{2}, \ldots, b_{(m-1) / 2}\right\}$ contains either $+x$ or $-x$ exactly once;

- narcissistic [2] if it is half-and-half and anti-directed, with the further property that its 2 -sequencing has $b_{i}=b_{m-i}$ for all $i=1,2, \ldots,(m-1) / 2$.

Some expositions include the zero element of $\mathbb{Z}_{m}$ in $\mathbf{b}$, as an extra element at the start, but we find this practice inconvenient, and we follow various precedents by not adopting it. For convenience we often write ' $\mathbb{Z}_{m}$ terrace' in place of 'terrace for $\mathbb{Z}_{m}$ '.

Terraces were originally defined by Bailey [8] for a general finite group $G$, but the general case does not concern us here.

Terraces are used in the construction of combinatorial designs used in statistical applications involving carry-over effects $[\mathbf{1}, \mathbf{8}]$ and neighbour effects. They are also implicit in the work of Ringel (e.g. [10, pp. 124, 129]) on graph embeddings and in some work on Hamiltonian double Latin squares [9].

Anderson and Preece [2-5] gave general constructions for 'power-sequence' terraces for $\mathbb{Z}_{m}$, where $m$ is odd. Each of these terraces can be partitioned into segments, one of which contains merely the zero element of $\mathbb{Z}_{m}$, whereas each other segment is either (a) a sequence of successive powers of an element of $\mathbb{Z}_{m}$ or (b) such a sequence multiplied throughout by a constant. Many of the sequences $x^{0}, x^{1}, \ldots, x^{s-1}$ of distinct elements are 'full-cycle' sequences such that $x^{s}=x^{0}$, but partial cycles are used too.

Anderson and Preece [6] showed that with $m=n-1$, where $n$ is odd, there are many ways in which power-sequences in $\mathbb{Z}_{n}$ can be used to arrange the elements of $\mathbb{Z}_{n} \backslash\{0\}$ in a sequence of distinct elements, usually in two or more segments, which becomes a terrace for $\mathbb{Z}_{m}$ when interpreted modulo $m$, with each element taking its value in the interval $[1, m]$. We now take the approach from [6] further, by moving on to $m=n-2$.

Each of our $\mathbb{Z}_{n-2}$ terraces consists of one or more segments, each comprising a sequence of distinct entries $\alpha_{1}, \alpha_{2}, \ldots, \alpha_{s}$, where $\alpha_{i+1}=2 \alpha_{i}$ or $2^{-1} \alpha_{i}$, modulo $n$ (not $n-2$ ), for $i=1,2, \ldots, s-1$. A segment may now be

(i) a full-cycle segment, with $\alpha_{2} / \alpha_{1}=\alpha_{1} / \alpha_{s}$;

(ii) a half-cycle segment, with $\alpha_{2} / \alpha_{1}=\alpha_{1} / \alpha_{2 s}$ (but $\alpha_{2} / \alpha_{1} \neq \alpha_{1} / \alpha_{s}$ );

(iii) a segment that would become a full cycle if the element $n-1$ were introduced at either end;

(iv) a segment that would become a half cycle if the element $n-1$ were introduced at one end; or

(v) a segment of irregular length.

In representations of terraces, we separate two segments that abut one another by a fence $\mid$. When a cycle is broken to form one or more segments, the difference between the two elements on each side of each break is 'lost' and becomes a 'missing difference'. We nevertheless usually need this difference to occur in the terrace, so we have to arrange that plus or minus this difference arises at a join between two adjacent segments; we then say that the missing difference is compensated for by a fence difference.

The sequence of elements in any one of our terraces $\left(a_{1}, a_{2}, \ldots, a_{n-2}\right)$ for $\mathbb{Z}_{n-2}$ is to be a permutation of the elements of $\mathbb{Z} \backslash\{0, n-1\}$, and the elements in it are to be written so that $0<a_{i}<n-1(i=1,2, \ldots, n-2)$. Thus with $n=7$ the sequence $\left(-2^{2},-2^{1}, 2^{0}, 2^{1}, 2^{2}\right)$ is evaluated in $\mathbb{Z}_{7}$ and written as $(3,5,1,2,4)$, which is a terrace for $\mathbb{Z}_{5}$. 
Let $\left(a_{1}, a_{2}, \ldots, a_{n-2}\right)$, written as just described, be any one of our terraces for $\mathbb{Z}_{n-2}$. Using subtraction modulo $n$, write

$$
d_{i}=a_{i+1}-a_{i}\left(0<d_{i}<n\right), \quad e_{i}=a_{i}-a_{i+1}\left(0<e_{i}<n\right)
$$

for $i=1,2, \ldots, n-3$. Likewise, using subtraction modulo $n-2$, write

$$
d_{i}^{* *}=a_{i+1}-a_{i}\left(0<d_{i}^{* *}<n\right), \quad e_{i}^{* *}=a_{i}-a_{i+1}\left(0<e_{i}^{* *}<n\right) .
$$

Now write $\mu_{i}=\min \left(d_{i}, e_{i}\right)$ and $\mu_{i}^{* *}=\min \left(d_{i}^{* *}, e_{i}^{* *}\right)$ for $i=1,2, \ldots, n-3$. Following [6] we call the values $\mu_{i}$ the $\mu$-differences for the terrace (from $\mu=\mathrm{mu}=$ 'minimum unsigned'), and we call the values $\mu_{i}^{* *}$ the corresponding $\mu^{* *}$-differences. For any particular value of $i$, the value $\mu_{i}^{* *}$ may equal $\mu_{i}$, or $\mu_{i}-1$, or $\mu_{i}-2$. We refer to the possibilities $\mu_{i}^{* *}=\mu_{i}-1$ and $\mu_{i}^{* *}=\mu_{i}-2$ as reduced differences. Returning to our example with $n=7$, the sequence $\left(-2^{2},-2^{1}, 2^{0}, 2^{1}, 2^{2}\right)$ has two segments, comprising two and three elements respectively. When it is evaluated as $(3,5,1,2,4)$, the consecutive entries 5 and 1 yield a reduced difference.

As each segment of any of our terraces is formed by successive multiplications by 2 or by $2^{-1}$, reduced differences can arise only (a) at the breaks between adjoining segments or (b) internally between the entries 1 and $(n+1) / 2$ or between the entries $n-1$ and $(n-1) / 2$. We exclude $n-1$ from all our sequences, so the second case in (b) does not arise. The first case in (b) gives us $\mu=(n-1) / 2$ and $\mu^{*}=(n-3) / 2$. Thus, if 1 is not the first or last entry in a segment, it must adjoin the entry $(n+1) / 2$, thereby producing the reduced difference $(n-3) / 2$ that compensates for the missing difference caused by having $(n-3) / 2$ at the end of a segment. This happens in the terraces produced via Theorems $2.5,3.2,4.1,4.3,4.4$ and 5.4.

In representations of our terraces, we replace the commas between entries by spaces. To aid the eye, we put a colon : at the start and end of each segment of type (ii) or (iv), and a scream ! at the start and end of a segment of irregular length. We put an asterisk $*$ at the start and end of a segment of type (iii) and at that end of a type (iv) segment at which the element $n-1$ would naturally lie. Thus our example with $n=7$ is written as

$$
*-2^{2}-2^{1} * \mid 2^{0} 2^{1} 2^{2} .
$$

If a segment starts with, say, an element $c$, and the successive elements are then $2 c, 2^{2} c, 2^{3} c, \ldots, d$, we sometimes find it helpful to write

$$
|c \stackrel{2}{\rightarrow}| \text { or }|c \stackrel{2}{\rightarrow} d| .
$$

Likewise, we use $\stackrel{2}{\leftarrow}$ when the successive multiplications are by $2^{-1}$ instead of 2 .

As in previous papers of ours, some of the theorems in this paper are for primes $n$ such that $\operatorname{ord}_{n}(2)<n-1$ but $\mathbb{Z}_{n} \backslash\{0\}=\langle 2,3\rangle$. Also, one of our theorems below requires $\langle 2,3\rangle$ in $\mathbb{Z}_{n}$ to contain precisely half of the elements of $\mathbb{Z}_{n} \backslash\{0\}$. In general, for a prime $n$, many different types of relationship between $\langle 2\rangle,\langle 2,3\rangle$ and $\mathbb{Z}_{n} \backslash\{0\}$ are possible. (For example for $n=23$ we have $\langle 2\rangle=\langle 3\rangle=\langle 2,3\rangle=$ the set of quadratic residues, modulo 23.) We need here comment only that for prime values of $n$, the relationship $\mathbb{Z}_{n} \backslash\{0\}=\langle 2,3$,$\rangle usually arises where any element x$ from $\mathbb{Z}_{n} \backslash\{0\}$ can be written in different inequivalent ways as a product of a power of 2 and a power of 3 . In the range $0<n<1,000$, the only prime $n$ with $\mathbb{Z}_{n} \backslash\{0\}$ equal to the direct 
product $\langle 2\rangle \times\langle 3\rangle$ is $n=683$ (for which we have failed to find a construction for this paper).

We need the following lemmata ( $n$ an odd prime power) for some of our theorems in $\S 2$ and $\S 3$.

LEMMA 1.1. If $u$ is an integer satisfying $0<u<n$, and $u$ and $3 u$ have the same parity when $3 u$ is evaluated, modulo $n$, in the interval $(0, n)$, then $u<n / 3$ or $u>2 n / 3$.

Proof. If $n / 3<u<2 n / 3$, then $3 u$ becomes $3 u-n$ when evaluated, modulo $n$, in the interval $(0, n)$. As $n$ is odd, the parity of $3 u-n$ then differs from that of $u$.

LEMMA 1.2. If $u$ is as in Lemma 1.1, then the fence difference in

$$
\ldots 3 u\left|2^{1} u 2^{2} u \ldots 2^{-1} u \quad u\right|
$$

compensates for the difference missing from the segment ending in $u$.

Proof. By Lemma 1.1, we have $u<n / 3$ or $u>2 n / 3$. If $u<n / 3$, the fence difference and the missing difference are both $u$. If $u>2 n / 3$, we have $3 u-2 n|2 u-n \ldots u|$. The fence difference is now $(2 u-n)-(3 u-2 n)=n-u$, and the missing difference too is $n-u$.

2. Theorems for specific small values of $(n-1) / \operatorname{ord}_{n}(2)$. We start with a theorem for any prime $n(>3)$ that has 2 as a primitive root.

THEOREM 2.1. Let $n$ be a prime, $n>3$, that has 2 as a primitive root. Then the following sequences, evaluated as described in $\S 1$, are terraces for $\mathbb{Z}_{n-2}$ :

(i) (narcissistic)

$$
: 2^{-1} 2^{-2} \ldots 2^{(n+1) / 2} *: \mid:: 2^{0} \quad 2^{1} \quad \ldots \quad 2^{(n-3) / 2}:
$$

(ii) (half-and-half and anti-directed)

as in (i) above, with the segments interchanged.

Proof.

(i) The difference between $2^{i+1}$ and $2^{i}$ is $2^{i}$, so the differences for the sequence are all members of $\mathbb{Z}_{n} \backslash\{0\}$ except $2^{-1}=(n+1) / 2=-(n-1) / 2,1$ and $2^{(n-3) / 2}=$ $-2^{-1}=(n-1) / 2$. At the fence we have $n-2 \mid 1$, which gives the compensating difference of 1 . So we lose the difference $(n-1) / 2$ twice as required, and the given sequence is indeed a terrace for $\mathbb{Z}_{n-2}$. As $2^{(n-1) / 2-i}=-2^{-i}$, the terrace is narcissistic.

(ii) Similar.

EXAMPLE 2.1. With $n=11$, Theorem 2.1 produces the following $\mathbb{Z}_{9}$ terrace of type (i):

$$
: 6379 *: \mid: 12485: \text {. }
$$

We now proceed to three theorems for primes $n$ such that $\operatorname{ord}_{n}(2)=(n-1) / 2$. 
THEOREM 2.2. Let $n$ be a prime, $n \equiv 7(\bmod 8)$, such that $\operatorname{ord}_{n}(2)=(n-1) / 2$. Then the following sequences, evaluated as described in $\S 1$, are terraces for $\mathbb{Z}_{n-2}$ :

(i) (narcissistic)

$$
\begin{array}{llllllllll}
*-1 & -2^{-2} & \ldots & -2^{1} & * & 2^{0} & 2^{1} & \ldots & 2^{-1}
\end{array}
$$

(ii) (half-and-half but not anti-directed)

$$
\text { as in (i) above, with the segments interchanged. }
$$

Proof. As $\operatorname{ord}_{n}(2)$ is odd, we have $-1 \notin\langle 2\rangle$, so $\mathbb{Z}_{n} \backslash\{0\}=\langle 2\rangle \cup-\langle 2\rangle$.

(i) The missing differences are $(n-1) / 2,1$ and $(n+1) / 2=-(n-1) / 2$, and the fence again has a reduced difference of 1 . The terrace is narcissistic as in Theorem 2.1.

(ii) Similar.

EXAMPLE 2.2. With $n=23$, Theorem 2.2 produces the following $\mathbb{Z}_{21}$ terrace of type (i):

$$
\text { * } 111720105147151921 * \text { | } 1224481691813 \quad 3 \quad 612 .
$$

TheOREM 2.3. Let $n$ be a prime, $n \equiv 1(\bmod 8)$, such that $\operatorname{ord}_{n}(2)=(n-1) / 2$. Suppose that $c$ is an integer satisfying $c \notin\langle 2\rangle$ and $0<c<(n-1) / 2$. Then the following sequence, evaluated as described in $\S 1$, is a terrace for $\mathbb{Z}_{n-2}$ :

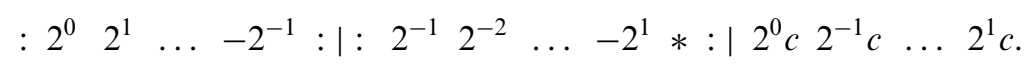

Proof. Here $\langle 2\rangle$ is the set of squares in $\mathbb{Z}_{n}$, and -1 is a square as $n \equiv 1(\bmod 8)$. So $-1 \in\langle 2\rangle$. As $x$ is a non-square if and only if $n-x$ is a non-square, we can find a non-square $c$ satisfying $0<c<(n-1) / 2$. The missing differences are $(n-1) / 2$, $(n+1) / 2=-(n-1) / 2,1$ and $c$. The first fence has difference 1 and the second has reduced difference $c$, and these compensate as required for missing differences.

EXAMPLE 2.3. With $n=17$, we can take $c=3,5,6$ or 7 in Theorem 2.3. With $c=3$ we obtain the $\mathbb{Z}_{15}$ terrace

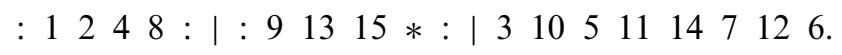

THEOREM 2.4. Let $n$ be a prime, $n \equiv 17(\bmod 24)$, such that $\operatorname{ord}_{n}(2)=(n-1) / 2$. Then the following sequences, evaluated as described in $\S 1$, are terraces for $\mathbb{Z}_{n-2}$ :

(i) (the two segments can be interchanged)

$$
*-2^{1}-2^{2} \ldots 2^{-1} * \mid-3 \cdot 2^{-1}-3 \cdot 2^{-2} \ldots-3 \cdot 2^{0}
$$

(ii)

$$
: 2^{0} 2^{1} \ldots-2^{-1}:\left|: 2^{-1} 2^{-2} \ldots-2^{1} *:\right|-3^{-1} \cdot 2^{2}-3^{-1} \cdot 2^{3} \ldots-3^{-1} \cdot 2^{1}
$$

(iii)

$$
: 2^{-1} 2^{-2} \ldots-2^{1} *:\left|: 2^{0} 2^{1} \ldots-2^{-1}:\right|-3^{-1} \cdot 2^{0}-3^{-1} \cdot 2^{1} \ldots-3^{-1} \cdot 2^{-1}
$$


Proof. As $n \equiv 17(\bmod 24)$, the elements 2 and -1 are squares in $\mathbb{Z}_{n}$ but 3 is not. So we can write $\mathbb{Z}_{n} \backslash\{0\}=\langle 2\rangle \cup 3\langle 2\rangle$, with $-1 \in\langle 2\rangle$.

(i) Here the missing differences are $(n-1) / 2,1,(n-3) / 2$. The difference at the first fence is 1 . The result follows, as $(n-3) / 2=-(n-1) / 2$ in $\mathbb{Z}_{n-2}$.

(ii) The missing differences are $(n-1) / 2$ (twice), 1 and $(n-2) / 3$. The first fence has difference 1 and the second has reduced difference $(n-2) / 3$.

(iii) Similar. The last missing difference $-3^{-1} \cdot 2^{-1}=(5 n-1) / 6=-(n+1) / 6$ is compensated for at the second fence, where the difference is $(2 n-1) / 3-(n-$ 1) $/ 2=(n+1) / 6$.

EXAMPLE 2.4. With $n=17$, Theorem 2.4 produces the following $\mathbb{Z}_{15}$ terrace of type (ii):

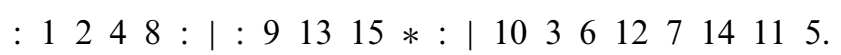

The next two theorems are for primes $n$ with $\operatorname{ord}_{n}(2)=(n-1) / 3$.

THEOREM 2.5. Let $n$ be a prime, $n \equiv 1(\bmod 6)$, such that $\operatorname{ord}_{n}(2)=(n-1) / 3$ and $\mathbb{Z}_{n} \backslash\{0\}=\langle 2,3\rangle$. Then the following sequence, evaluated as described in $\S 1$, is a terrace for $\mathbb{Z}_{n-2}$ :

$$
\begin{aligned}
& -3^{-1} \cdot 2^{-1}-3^{-1} \cdot 2^{-2} \ldots-3^{-1} \cdot 2^{0}
\end{aligned}
$$

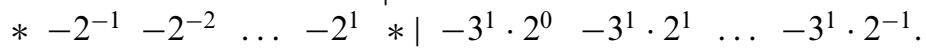

Proof. We have $\mathbb{Z}_{n} \backslash\{0\}=-\langle 2\rangle \cup-3\langle 2\rangle \cup-3^{-1}\langle 2\rangle$, where $-1 \in\langle 2\rangle$. Checking the differences is routine.

Note 2.5. In the range $3<n<1000$, Theorem 2.5 covers $n=43,109,157,229$, 277, 283, 691, 733, 739 and 811 .

EXAMPLE 2.5. With $n=43$, Theorem 2.5 produces the following $\mathbb{Z}_{41}$ terrace:

$$
\begin{array}{lllll|llllllllllll|lllll}
7 & 25 & \ldots & 28 & 14 & * & 21 & 32 & \ldots & 2 & 1 & 22 & 11 & \ldots & 39 & 41 & * & 40 & 37 & \ldots & 10 & 20 .
\end{array}
$$

A generalisation of our next theorem, Theorem 2.6, could readily be provided to cover Theorem 2.7 too, but we keep the two cases separate for clarity.

THEOREM 2.6. Let $n$ be a prime, $n \equiv 1(\bmod 6)$, such that $\operatorname{ord}_{n}(2)=(n-1) / 3$. Suppose that $c_{1}$ and $c_{2}$ are integers such that $c_{2}=3 c_{1}-4$, where $c_{1}$ is odd, with $0<$ $c_{1}<n / 3$ and $\mathbb{Z}_{n} \backslash\{0\}=\langle 2\rangle \cup c_{1}\langle 2\rangle \cup c_{2}\langle 2\rangle$. Then the following sequence, evaluated as described in $\S 1$, is a terrace for $\mathbb{Z}_{n-2}$ :

$$
: 2^{0} \quad 2^{1} \quad \ldots \quad-2^{-1}:\left|: 2^{-1} 2^{-2} \ldots 2^{1} *:\right| 2^{-2} \delta c_{2} \stackrel{2}{\leftarrow} \mid \delta c_{1} \stackrel{2}{\rightarrow},
$$

where $\delta= \pm 1$ accordingly as $n \equiv \pm c_{1}(\bmod 4)$.

Proof. The first three missing differences are $(n-1) / 2$ (twice) and 1 , with 1 being compensated for at the first fence.

(i) First suppose that $n \equiv c_{1}(\bmod 4)$, and take $\delta=1$. Then $c_{2} \equiv-n(\bmod 4)$, and the remaining missing differences are $\left(n+c_{2}\right) / 4$ (which is less than $\left.n / 2\right)$ and $\left(n+c_{1}\right) / 2=-\left(n-c_{1}\right) / 2$. The second and final fences give reduced differences $\left(n+c_{2}\right) / 4$ and $c_{1}+\left(n-2-\left(n+c_{2}\right) / 2\right)=\left(n-c_{1}\right) / 2$ respectively. 
(ii) Now suppose that $n \equiv-c_{1}(\bmod 4)$, so that $c_{2} \equiv n(\bmod 4)$ and $\delta=-1$. The last two missing differences are now $\left(n-c_{2}\right) / 4$ and $\left(n-c_{1}\right) / 2$, and these are similarly shown to be compensated for at the fences.

Note 2.6. In the range $3<n<1,000$, Theorem 2.6 covers $n$-values as follows, where the smallest possible value of $c_{1}$ is listed for each $n$ :

$\begin{array}{rrrrrrrrrrrrrrr}n & 43 & 109 & 157 & 229 & 277 & 283 & 307 & 499 & 643 & 691 & 733 & 739 & 811 & 997 \\ \min c_{1} & 9 & 5 & 13 & 3 & 3 & 3 & 11 & 5 & 7 & 15 & 9 & 9 & 3 & 7\end{array}$

EXAMPLE 2.6. With $n=109$, we can take $c_{1}=5,11,13$ or 31 in Theorem 2.6. With $c_{1}=5$ we obtain the following terrace for $\mathbb{Z}_{107}$ :

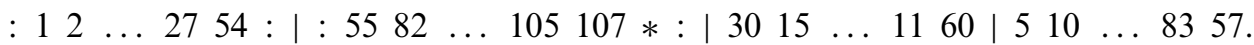

We now extend the idea used in Theorem 2.6 from $\operatorname{ord}_{n}(2)=(n-1) / 3$ to $\operatorname{ord}_{n}(2)=$ $(n-1) / 4$.

THEOREM 2.7. Let $n$ be a prime, $n \equiv 1(\bmod 8)$, such that $\operatorname{ord}_{n}(2)=(n-1) / 4$. Suppose that $c_{1}, c_{2}$ and $c_{3}$ are integers such that $c_{i+1}=3 c_{i}-4(i=1,2)$, where $c_{1}$ is odd, with $0<c_{1}<n / 9$ and $\mathbb{Z}_{n} \backslash\{0\}=\langle 2\rangle \cup \bigcup_{i=1}^{3} c_{i}\langle 2\rangle$. Then the following sequence, evaluated as described in $\S 1$, is a terrace for $\mathbb{Z}_{n-2}$ :

$: 2^{0} \quad 2^{1} \ldots 2^{-1}:\left|: 2^{-1} 2^{-2} \ldots 2^{1} *:\right| 2^{-2} \delta c_{3} \stackrel{2}{\leftarrow}\left|\delta c_{2} \stackrel{2}{\rightarrow}\right| \delta c_{1} \stackrel{2}{\rightarrow}$,

where $\delta= \pm 1$ according as $n \equiv \mp c_{1}(\bmod 4)$.

Proof. Similar to that of Theorem 2.6.

Note 2.7. In the range $3<n<1,000$, Theorem 2.7 covers $n$-values as follows:

$\begin{array}{rrrrrrr}n & 113 & 281 & 353 & 577 & 593 & 617 \\ \min c_{1} & 3 & 9 & 33 & 5 & 17 & 35\end{array}$

ExAMPLE 2.7. With $\left(n, c_{1}\right)=(113,3)$, Theorem 2.7 yields the following terrace for $\mathbb{Z}_{111}$ :

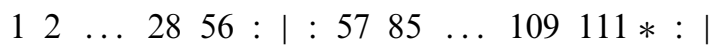

$$
\begin{aligned}
& \begin{array}{lllll|lllll|lllll}
31 & 72 & \ldots & 11 & 62 & 5 & 10 & \ldots & 86 & 59 & 3 & 6 & \ldots & 29 & 58 .
\end{array}
\end{aligned}
$$

We now end this section of the paper with three theorems specifically for values of $n$ with $\operatorname{ord}_{n}(2)=(n-1) / 6$. For the first two of these theorems, $\operatorname{ord}_{n}(2)$ is odd, whereas for the third it is even.

THEOREM 2.8. Let $n$ be a prime, $n \equiv 7(\bmod 24)$, such that $\operatorname{ord}_{n}(2)=(n-1) / 6$. Suppose that $\mathbb{Z}_{n} \backslash\{0\}=\langle 2,3\rangle$ and that $3 \cdot 11 \in\langle 2\rangle$. Then the following sequence, evaluated as described in $\S 1$, is a terrace for $\mathbb{Z}_{n-2}$.

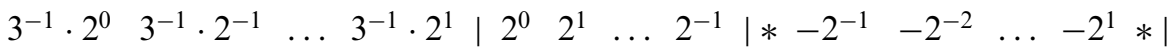

$$
\begin{aligned}
& 3^{2} \cdot 2^{-3} \quad 3^{2} \cdot 2^{-4} \quad \ldots 3^{2} \cdot 2^{-2}\left|3 \cdot 2^{-1} \quad 3 \cdot 2^{0} \ldots 3 \cdot 2^{-2}\right| \\
& 11 \cdot 3^{-1} \cdot 2^{-1} 11 \cdot 3^{-1} \cdot 2^{0} \ldots 11 \cdot 3^{-1} \cdot 2^{-2} \text {. }
\end{aligned}
$$


Proof. As $n \equiv 7(\bmod 24)$, the element 2 is a square in $\mathbb{Z}_{n}$ but -1 and 3 are not. Also, $-1 \in 3^{3}\langle 2\rangle$ and $11 \cdot 3^{-1} \in 3^{-2}\langle 2\rangle=3^{4}\langle 2\rangle$ as $3 \cdot 11 \in\langle 2\rangle$. Thus $11 \cdot 3^{-1}\langle 2\rangle=3^{4}\langle 2\rangle$.

The missing differences are $(2 n+1) / 3=-(n-1) / 3, \quad(n+1) / 2=-(n-1) / 2$, $(n-1) / 2,1,(n+9) / 8,(3 n+3) / 4=-(n-3) / 4$ and $(7 n+11) / 12=-(5 n-11) / 12$. The fence differences are $(n+2) / 3-1=(n-1) / 3,1, \quad(n+9) / 8$ (reduced), $(n+3) / 2-(n+9) / 4=(n-3) / 4$ and $(n+11) / 6+(n-2-(3 n+3) / 4)=(5 n-11) / 12$ (reduced).

Note 2.8. In the range $3<n<1,000$, Theorem 2.8 covers only $n=31$ and 223 . The $n$-values 439, 727 and 919 are not covered as, for each of them, $3 \in-\langle 2\rangle$.

EXAMPLE 2.8. With $n=31$, Theorem 2.8 produces the following terrace for $\mathbb{Z}_{29}$ :

$$
\begin{array}{rrrrrrrrrrrrrrrrrrr}
21 & 26 & 13 & 22 & 11 & \mid & 1 & 2 & 4 & 8 & 16 & \mid & * & 15 & 23 & 27 & 29 & * & \text { | } \\
5 & 18 & 9 & 20 & 10 & \mid & 17 & 3 & 6 & 12 & 24 & \mid & 7 & 14 & 28 & 25 & 19 . & &
\end{array}
$$

THEOREM 2.9. Let $n$ be a prime, $n \equiv 7(\bmod 24)$, such that $\operatorname{ord}_{n}(2)=(n-1) / 6$. Suppose that $3 \in-\langle 2\rangle$ and that $c$ and $d$ are integers such that $c$ and $3 c$ are both even, $d$ and $3^{-1} d$ are both odd and $\mathbb{Z}_{n} \backslash\{0\}=\langle 2,-1\rangle \cup c\langle 2,-1\rangle \cup d\langle 2,-1\rangle$. Then $c<n / 3$ or $c>2 n / 3$, and the sequence

$$
\begin{aligned}
& 2^{0} 2^{1} \ldots 2^{-1}\left|*-2^{-1}-2^{-2} \ldots 2^{1} *\right| \\
& 3^{1} \cdot 2^{-1} c \stackrel{2}{\leftarrow}\left|3^{0} \cdot 2 c \stackrel{2}{\rightarrow}\right| 3^{0} \cdot 2 d \stackrel{2}{\rightarrow} \mid 3^{-1} \cdot 2 d \stackrel{2}{\rightarrow},
\end{aligned}
$$

evaluated as described in $\S 1$, is a terrace for $\mathbb{Z}_{n-2}$ provided that

(i) either $c<n / 3$ and $c=3 d+2-n$

(ii) or $c>2 n / 3$ and $c=3 d-2-n$.

Proof. That $c<n / 3$ or $c>2 n / 3$ follows from Lemma 1.1 .

The missing differences are $(n-1) / 2$ (twice), $1,3 c / 2, c, d$ and $3^{-1} d$. The difference at the first fence is 1 . The second fence has the reduced difference $3 c / 2$ if $c<n / 3$ (as we then have $3 c / 2<n / 2$ ); on the other hand, if $c>2 n / 3$, we have $n-2 \mid 3 c / 2-n$, where $3 c / 2-n<n / 2$, so the reduced difference is then $3 c / 2-n$.

The third fence has $3 c \mid 2 c$, which yields the difference $c$ if $c<n / 3$. If $c>2 n / 3$, however, we have $3 c-2 n \mid 2 c-n$, for which the difference is $n-c$.

At the fourth fence we have $c \mid 2 d$, and we now show that this yields a reduced difference of $d$ (or $n-d)$ ). First suppose that $c<n / 3$. Then $d=(n+c-2) / 3$, and we have $d<(4 n-6) / 9<(n-2) / 2$. As $2 d-c=n-d-2>n / 2$, the fence has the reduced difference $c+(n-2-2 d)=d$. Now suppose that $c>2 n / 3$. Then $d=(n+$ $c+2) / 3>(5 n+6) / 9$. We have $c \mid 2 d-n$, where $c-(2 d-n)=d-2>n / 2$, so the fence has the reduced difference $(2 d-n)+(n-2-c)=n-d$.

At the final fence we have $d \mid 3^{-1} \cdot 2 d$, which may be written as $3 u \mid 2 u$, where the elements $u=3^{-1} \cdot d$ and $3 u$ are both odd. The end of the sequence is $3 u \mid 2 u \ldots u$, and by Lemma 1.2, the fence difference is $u=3^{-1} \cdot d$.

Note 2.9. In the range $3<n<1,000$, Theorem 2.9 covers only $n=439,727$ and 919.

EXAMPLES 2.9. With $n=439$ we can take $(c, d)=(22,153),(306,249),(342,261)$, $(360,267)$ or $(396,279)$ in Theorem 2.9 . With $(c, d)=(306,249)$ we obtain the 
following $\mathbb{Z}_{437}$ terrace:

$$
\begin{aligned}
& \begin{array}{llllll|lllllll|lllll|}
1 & 2 & \ldots & 110 & 220 & * & 219 & 329 & \ldots & 435 & 437 & * & 20 & 10 & \ldots & 80 & 40
\end{array} \mid \\
& \begin{array}{lllll|lllll|llllll}
173 & 346 & \ldots & 153 & 306 & 59 & 118 & \ldots & 344 & 249 & 166 & 332 & \ldots & 26183 .
\end{array}
\end{aligned}
$$

With $n=727$ we can take $(c, d)=(666,465)$, and with $n=919$ we can take $(c, d)=$ $(738,553)$.

THEOREM 2.10. Let $n$ be a prime, $n \equiv 1(\bmod 12)$, such that $\operatorname{ord}_{n}(2)=6$. Suppose that $\langle 2,3\rangle$ comprises half of $\mathbb{Z}_{n} \backslash\{0\}$ and that $c$ and $d$ are integers such that $c, 3^{1} c$ and $3^{2} c$ are all even and $d \in 3\langle 2\rangle$. Then the sequence

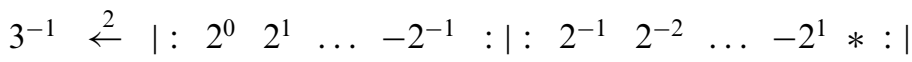

$$
\begin{aligned}
& 3^{2} \cdot 2^{-1} c \stackrel{2}{\leftarrow}\left|3^{1} \cdot 2 c \stackrel{2}{\rightarrow}\right| 3^{0} \cdot 2 c \stackrel{2}{\rightarrow} \mid 3^{0} \cdot 2 d \stackrel{2}{\rightarrow},
\end{aligned}
$$

evaluated as described in $\S 1$, is a terrace for $\mathbb{Z}_{n-2}$ provided that

either (i) $c<n / 3$ and $c=3 d-n+2$

or (ii) $c>2 n / 3$ and $c=3 d-n-2$.

Proof. Here $\operatorname{ord}_{n}(2)$ is even, so $-1 \in\langle 2\rangle$. That $c<n / 3$ or $c>2 n / 3$ follows from Lemma 1.1.

The only non-trivial part of the proof is to show that the elements $c \mid 2 d$ at the final fence yield a reduced difference that compensates for the missing difference $d$ (or $n-d$ ). But this follows as for Theorem 2.9.

Note 2.10. In the range $3<n<1,000$, Theorem 2.10 covers only $n=433$ and 457 .

EXAMPLES 2.10. With $n=433$ Theorem 2.10 yields a $\mathbb{Z}_{431}$ terrace with $(c, d)=$ $(336,257)$. With $n=457$ Theorem 2.10 yields a $\mathbb{Z}_{455}$ terrace with $(c, d)=(426,295)$.

3. General constructions, $\operatorname{ord}_{n}(2)=(n-1) / k$. The first theorem in this section provides a construction for narcissistic terraces.

THEOREM 3.1. Let $n$ be a prime such that $\mathbb{Z}_{n} \backslash\{0\}=\langle 2,3\rangle$ and such that $\operatorname{ord}_{n}(2)$ is even and equal to $(n-1) / k$, where $k>1$. Suppose that the integers $3^{0}$. $2^{-1}, 3^{-1} \cdot 2^{-1}, \ldots, 3^{-(k-1)} \cdot 2^{-1}$ all have the same parity. Then the following sequence, evaluated as described in $\S 1$, is a narcissistic terrace for $\mathbb{Z}_{n-2}$ :

$$
\begin{gathered}
:(-1)^{k-1} 3^{-(k-1)} \cdot 2^{-1} \stackrel{2}{\leftarrow}:\left|:(-1)^{k-2} 3^{-(k-2)} \cdot 2^{-1} \stackrel{2}{\leftarrow}:\right| \cdots \mid \\
:-3^{-1} \cdot 2^{-1} \stackrel{2}{\leftarrow}:\left|: 3^{0} \cdot 2^{-1} 3^{0} \cdot 2^{-2} \ldots 3^{0} \cdot\left(-2^{1}\right) *:\right|: 3^{0} \cdot 2^{0} \stackrel{2}{\rightarrow}: \mid \\
:(-1)^{1} 3^{-1} \cdot 2^{0} \stackrel{2}{\rightarrow}:\left|:(-1)^{2} 3^{-2} \cdot 2^{0} \stackrel{2}{\rightarrow}:\right| \cdots \mid:(-1)^{k-1} 3^{-(k-1)} \cdot 2^{0} \stackrel{2}{\rightarrow}: .
\end{gathered}
$$

Proof. As $\operatorname{ord}_{n}(2)$ is even we have $-1 \in\langle 2\rangle$. The first $k-1$ segments are of the form $-u \ldots 2 u \mid 3 u$, where $u$ and $3 u$, evaluated in [1,n-1], have the same parity. By Lemma 1.1, we have $u<n / 3$ or $u>2 n / 3$. If $0<u<n / 3$, then the missing difference $u$ is compensated for by the fence difference. If $2 n / 3<u<n$, the missing difference is $n-u$, and the fence elements $(2 u-n) \mid(3 u-2 n)$ give the compensating difference $n-u$.

The next segment is $\left|2^{-1} \ldots-2 *\right|$, which has missing differences $(n-1) / 2$ and 1 ; the 1 is compensated for at the next fence. The next segment too has missing 
difference $(n-1) / 2$. Thereafter, the format is $3 u \mid 2 u \ldots-u$, and the missing and fence differences again compensate. So we lose $(n-1) / 2$ twice, as required.

Note 3.1. Theorem 3.1 covers all primes $n \equiv 17(\bmod 24)$ with $\operatorname{ord}_{n}(2)=(n-1) / 2$, and, subject to the restriction $\mathbb{Z}_{n} \backslash\{0\}=\langle 2,3\rangle$, it covers all primes $n \equiv 1$ or $7(\bmod 18)$ with $\operatorname{ord}_{n}(2)=(n-1) / 3$. In the range $3<n<1,000$, the coverage is as follows:

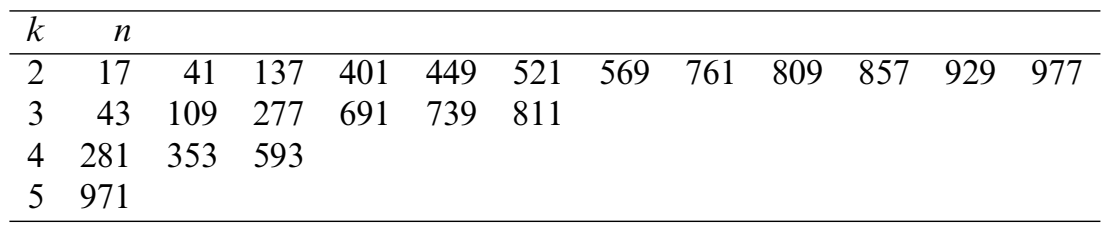

EXAmple 3.1. With $(n, k)=(43,3)$, Theorem 3.1 yields the following terrace for $\mathbb{Z}_{41}$ :

$$
\begin{aligned}
& \text { : } \begin{array}{llllllll|llllllll|}
12 & 6 & 3 & 23 & 33 & 38 & 19 & : & 25 & 34 & 17 & 30 & 15 & 29 & :
\end{array} \\
& \text { : } 221127353941 * \text { : | : } 12448163221 \text { : | } \\
& \text { : } 1428132691836 \text { : |: : } 2451020403731 \text { :. }
\end{aligned}
$$

THEOREM 3.2. Let $n$ be a prime such that $\mathbb{Z}_{n} \backslash\{0\}=\langle 2,3\rangle$ and such that $\operatorname{ord}_{n}(2)$ is even and equal to $(n-1) / k$, where $k>2$. Suppose that $c$ is an integer satisfying $0<c<(n-1) / 2$ and $c \in 3^{-1}\langle 2\rangle$. Suppose further that the integers $3^{0} \cdot 2 c, 3^{-1} \cdot 2 c, \ldots$, $3^{-(k-3)} \cdot 2 c$ are all even. Then the following sequence, evaluated as described in $\S 1$, is a terrace for $\mathbb{Z}_{n-2}$ :

$$
\begin{aligned}
& -3 \stackrel{2}{\rightarrow}\left|*-2^{-1}-2^{-2} \ldots-2^{1} *\right| \\
& 3^{0} \cdot c \stackrel{2}{\leftarrow}\left|3^{-1} \cdot 2^{2} c \stackrel{2}{\rightarrow}\right| 3^{-2} \cdot 2^{2} c \stackrel{2}{\rightarrow}|\cdots| 3^{-(k-3)} \cdot 2^{2} c \stackrel{2}{\rightarrow} .
\end{aligned}
$$

Proof. The first three segments have missing differences $(n-3) / 2,(n-1) / 2,1$ and $c$. The last two of these are compensated for at the first two fences. The remaining segments are dealt with as in the proof of Theorem 3.1, and the result follows, as $(n-3) / 2 \equiv-(n-1) / 2$, modulo $n-2$.

Note 3.2. In the range $3<n<1,000$, Theorem 3.2 covers $n$-values as follows (for $n=397$ we can take $c=135$, and for each of the other $n$-values we can take $c=3^{k-1}$ ):

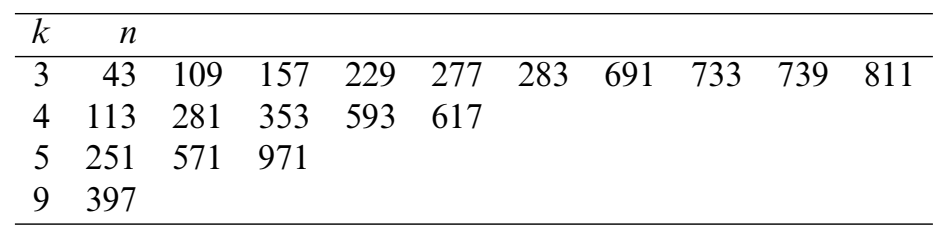

The theorem fails, in a 'near miss', to provide a terrace with $(n, k)=(641,10)$.

EXAmple 3.2. With $(n, k)=(43,3)$ and $c=7$, Theorem 3.2 yields the following terrace for $\mathbb{Z}_{41}$ :

\begin{tabular}{llllllll|llllllll|lllllll}
40 & 37 & 31 & $\ldots$ & 5 & 10 & 20 & $*$ & 21 & 32 & 16 & $\ldots$ & 35 & 39 & 41 & $*$ & 7 & 25 & 34 & $\ldots$ & 13 & 28 & 14.
\end{tabular} 
THEOREM 3.3. Let $n$ be a prime, $n \equiv 1$ or $13(\bmod 18)$, such that $\mathbb{Z}_{n} \backslash\{0\}=\langle 2,3\rangle$ and such that $\operatorname{ord}_{n}(2)$ is even and equal to $(n-1) / k$, where $k>2$. Suppose that the integers $3^{-1}, 3^{-2}, \ldots, 3^{-(k-1)}$ are all odd. Then the following sequence, evaluated as described in $\S 1$, is a terrace for $\mathbb{Z}_{n-2}$ :

$$
\begin{aligned}
& 3^{-(k-1)} \stackrel{2}{\leftarrow}\left|3^{-(k-2)} \stackrel{2}{\leftarrow}\right| \cdots\left|3^{-1} \stackrel{2}{\leftarrow}\right|
\end{aligned}
$$

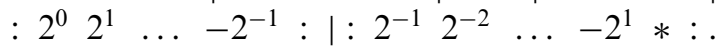

Proof. The congruence conditions ensure that $3^{-1}$ and $3^{-2}$ are both odd. The first $k-1$ segments are each of the form $x \ldots 2 x \mid 3 x$, where $x$ and $3 x$ are odd, and by Lemma 1.2 this ensures that the fence differences compensate for the missing differences. Thereafter, the missing differences are $(n-1) / 2$ (twice) and 1 ; the fence difference is 1 .

Note 3.3. In the range $3<n<1,000$, Theorem 3.3 covers $(n, k)=(109,3),(157,3)$, $(229,3),(283,3),(571,5),(733,3),(739,3)$ and $(811,3)$.

EXAMPLE 3.3. With $(n, k)=(109,3)$, Theorem 3.3 yields the following terrace for $\mathbb{Z}_{107}$ :

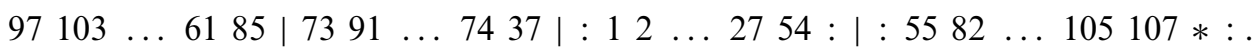

THEOREM 3.4. Let $n$ be a prime, $n \equiv 1(\bmod 6)$, such that $\mathbb{Z}_{n} \backslash\{0\}=\langle 2,3\rangle$ and such that $\operatorname{ord}_{n}(2)$ is even and equal to $(n-1) / k$, where $k>2$. Suppose that $c$ is an integer satisfying $0<c<(n-1) / 2$ and $c \in 3^{-2}\langle 2\rangle$ [not $3^{-1}\langle 2\rangle$ as in previous theorems]. Suppose further that the integers $3^{0} \cdot 2 c, 3^{-1} \cdot 2 c, \ldots, 3^{-(k-3)} \cdot 2 c$ are all even. Then $k$ is odd, and the following sequence, evaluated as described in $\S 1$, is a terrace for $\mathbb{Z}_{n-2}$ :

$$
\begin{gathered}
3^{-1} \stackrel{2}{\leftarrow}\left|: 2^{0} 2^{1} \ldots 2^{-1}:\right|: 2^{-1} 2^{-2} \ldots 2^{-1} *: \mid \\
3^{0} \cdot c \stackrel{2}{\leftarrow}\left|3^{-1} \cdot 2^{2} c \stackrel{2}{\rightarrow}\right| 3^{-2} \cdot 2^{2} c \stackrel{2}{\rightarrow}|\cdots| 3^{-(k-3)} \cdot 2^{2} c \stackrel{2}{\rightarrow} .
\end{gathered}
$$

Proof. Suppose that $k$ is even, with $k=2 h$. Then $(n-1) / k=(n-1) / 2 h$ is even, so $n \equiv 1(\bmod 4)$. But $n \equiv 1(\bmod 6)$, so $n \equiv 1(\bmod 12)$. But then 3 is a square in $\mathbb{Z}_{n}$, and so 2 is not; thus $n \equiv 13(\bmod 24)$, and $(n-1) / 4$ is odd. But $-1=2^{(n-1) / 4 h}$ is a square in $\mathbb{Z}_{n}$, as $n \equiv 1(\bmod 4)$, and 2 is not, so $(n-1) / 4 h$ is even. Thus $(n-1) / 4$ is even - which gives us a contradiction.

The rest of the proof is similar to the proof of Theorem 3.3.

Note 3.4. If for some $n$ satisfying $n \equiv 1(\bmod 6)$, Theorem 3.2 provides a $\mathbb{Z}_{n-2}$ terrace with $c=3 \gamma$, then Theorem 3.4 yields a $\mathbb{Z}_{n-2}$ terrace with $c=\gamma$. In the range $3<n<1,000$, Theorem 3.4 covers $n$-values as follows (for $n=397$ we can take $c=45$, and for each of the other $n$-values we can take $\left.c=3^{k-2}\right)$ :

\begin{tabular}{r|rrrrrrrrrrrr}
\hline$n$ & 43 & 109 & 157 & 229 & 277 & 283 & 397 & 571 & 691 & 733 & 739 & 811 \\
$k$ & 3 & 3 & 3 & 3 & 3 & 3 & 9 & 5 & 3 & 3 & 3 & 3 \\
\hline
\end{tabular}


EXAmple 3.4. For $(n, k)=(43,3)$ we can take $c=3,5,6,10,12,19$ or 20 in Theorem 3.4. Taking $c=3$ yields the $\mathbb{Z}_{41}$ terrace

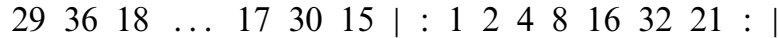

$$
\begin{aligned}
& \text { : } 221127353941 * \text { : | } \begin{array}{llllllll}
3 & 23 & 33 & \ldots & 24 & 12 & 6 .
\end{array}
\end{aligned}
$$

THEOREM 3.5. Let $n$ be a prime, $n \equiv 1(\bmod 24)$, such that $\langle 2,3\rangle$ contains half of the elements of $\mathbb{Z}_{n} \backslash\{0\}$ and such that $\operatorname{ord}_{n}(2)$ is even and equal to $(n-1) / 2 h$ where $h>1$. Suppose that $c$ is an integer satisfying $0<c<(n-1) / 2$ and $c \notin\langle 2,3\rangle$. Suppose further that the integers $3^{-1}, 3^{-2}, \ldots, 3^{-(h-1)}$ are all odd and that the integers $3^{0} \cdot 2 c, 3^{-1}$. $2 c, \ldots, 3^{-(h-1)} \cdot 2 c$ are all even. Then the following sequences, evaluated as described in $\S 1$, are terraces for $\mathbb{Z}_{n-2}$ :

(i)

$$
\begin{aligned}
& 3^{-(h-1)} \stackrel{2}{\leftarrow}\left|3^{-(h-2)} \stackrel{2}{\leftarrow}\right| \cdots\left|3^{-1} \stackrel{2}{\leftarrow}\right|
\end{aligned}
$$

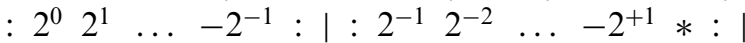

$$
\begin{aligned}
& 3^{0} \cdot c \stackrel{2}{\leftarrow}\left|3^{-1} \cdot 2^{2} c \stackrel{2}{\rightarrow}\right| 3^{-2} \cdot 2^{2} c \stackrel{2}{\rightarrow}|\cdots| 3^{-(h-1)} \cdot 2^{2} c \stackrel{2}{\rightarrow}
\end{aligned}
$$

(ii)

as in (i) above, save that the first $h-1$ segments are negated.

Proof. The proof is similar to that of Theorem 3.4. At the start the segments form the pattern $x \ldots 2 x \mid 3 x$, and at the end they form the pattern $3 x \mid 2 x \ldots x$. The condition $n \equiv 1(\bmod 24)$ is necessary, as explained in the proof of Theorem 5.6 of [7].

Note 3.5: In the range $3<n<1,000$, Theorem 3.5 covers only $(n, h)=(433,3)$. The $n$-values given by $(n, h)=(241,5),(457,3)$ and $(673,7)$ fail, as they all have $3^{-2}$ even.

EXAmple 3.5. For $(n, h)=(433,3)$, we can take $c=5$ in Theorem 3.5 to obtain the following $\mathbb{Z}_{431}$ terrace of type (i):

$$
\begin{aligned}
& \begin{array}{lllll|lllll|}
385 & 409 & \ldots & 241 & 337 & 289 & 361 & \ldots & 290 & 145
\end{array}
\end{aligned}
$$

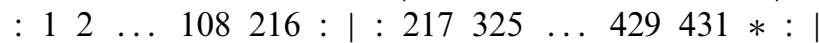

$$
\begin{aligned}
& \begin{array}{llllll|lllll|lllll}
5 & 219 & \ldots & 20 & 10 & 151 & 302 & \ldots & 146 & 292 & 339 & 245 & \ldots & 193 & 386 .
\end{array}
\end{aligned}
$$

We now turn to theorems for situations in which $\operatorname{ord}_{n}(2)$ is odd.

THEOREM 3.6. Let $n$ be a prime such that $\mathbb{Z}_{n} \backslash\{0\}=\langle 2,3\rangle$ and such that $\operatorname{ord}_{n}(2)$ is odd and equal to $(n-1) / 2 h$, where $h>1$. Suppose that the integers $3^{0} \cdot 2^{-1}$, $3^{-1} \cdot 2^{-1}, \ldots, 3^{-(h-1)} \cdot 2^{-1}$ all have the same parity. Then $h>2$, and the following sequence, evaluated as described in $\S 1$, is a narcissistic terrace for $\mathbb{Z}_{n-2}$ :

$$
\begin{gathered}
-3^{-(h-1)} \cdot 2^{-1} \quad \stackrel{2}{\leftarrow}\left|-3^{-(h-2)} \cdot 2^{-1} \stackrel{2}{\leftarrow}\right| \cdots\left|-3^{-1} \cdot 2^{-1} \stackrel{2}{\leftarrow}\right| \\
*-2^{-1} \quad-2^{-2} \quad \ldots \quad-2^{1} *\left|2^{0} \stackrel{2}{\rightarrow}\right| \\
3^{-1} \stackrel{2}{\rightarrow}\left|3^{-2} \stackrel{2}{\rightarrow}\right| \cdots \mid 3^{-(h-1)} \stackrel{2}{\rightarrow} .
\end{gathered}
$$


Proof. Suppose that $h=2$. Then $\operatorname{ord}_{n}(2)=(n-1) / 4$. As $n \equiv 1(\bmod 4)$ and 2 is a square in $\mathbb{Z}_{n}$, we have $n \equiv 1(\bmod 8)$. But then $(n-1) / 4$ is even - which gives us a contradiction. So $h>2$.

We next observe that $-1 \in 3^{h}\langle 2\rangle$ and that

$$
\mathbb{Z}_{n} \backslash\{0\}=\left\{\bigcup_{i=0}^{h-1} 3^{i}\langle 2\rangle\right\} \cup\left\{\bigcup_{i=0}^{h-1}-3^{i}\langle 2\rangle\right\} .
$$

The checking of differences is standard.

Note 3.6. In the range $3<n<1,000$, Theorem 3.6 covers only $(n, h)=(89,4)$ and $(223,3)$.

EXAMPLE 3.6. With $(n, h)=(89,4)$, Theorem 3.6 yields the $\mathbb{Z}_{87}$ terrace

$$
\begin{aligned}
& \begin{array}{lllllllll|llllllllllll}
28 & 14 & \ldots & 56 & 84 & 42 & \ldots & 79 & 74 & 37 & \ldots & 59 & * & 44 & 22 & \ldots & 87 & *
\end{array} \\
& \begin{array}{llll|llll|llll|llll}
1 & 2 & \ldots & 45 & 30 & 60 & \ldots & 15 & 10 & 20 & \ldots & 5 & 33 & 66 & \ldots & 61 .
\end{array}
\end{aligned}
$$

THEOREM 3.7. Let $n$ be a prime, $n \equiv 1(\bmod 6)$, such that $\mathbb{Z}_{n} \backslash\{0\}=\langle 2,3\rangle$ and such that $\operatorname{ord}_{n}(2)$ is odd and equal to $(n-1) / 2 h$, where $h>2$. Suppose that $c$ is an integer satisfying $0<c<(n-1) / 2$ and $c \in \pm 3^{h-1}\langle 2\rangle$. Suppose further that the integers $3^{-1}$, $3^{-2}, \ldots, 3^{-(h-1)}$ are all odd and that the integers $3^{0} \cdot 2 c, 3^{-1} \cdot 2 c, \ldots, 3^{-(h-2)} \cdot 2 c$ are all even. Then the following sequences, evaluated as described in $\S 1$, are half-and-half terraces for $\mathbb{Z}_{n-2}$ :

(i) If $c \in+3^{h-1}\langle 2\rangle$,

$$
\begin{aligned}
& 3^{-(h-1)} \stackrel{2}{\leftarrow}\left|3^{-(h-2)} \stackrel{2}{\leftarrow}\right| \ldots\left|3^{-1} \stackrel{2}{\leftarrow}\right| \\
& 3^{0} \cdot c \stackrel{2}{\leftarrow}\left|3^{-1} \cdot 2^{2} c \stackrel{2}{\rightarrow}\right| 3^{-2} \cdot 2^{2} c \stackrel{2}{\rightarrow}|\cdots| 3^{-(h-2)} \cdot 2^{2} c \stackrel{2}{\rightarrow} \text {. }
\end{aligned}
$$

(ii) If $c \in-3^{h-1}\langle 2\rangle$,

as in (i) above, save that the first $h-1$ segments are negated.

Proof. The first $h-2$ segments form the pattern $x \ldots 2 x \mid 3 x$, where $x$ and $3 x$ have the same parity, whereas the last $h-2$ segments form the pattern $3 y \mid 2 y \ldots y$, where $3 y$ and $y$ have the same parity. So the differences behave as in previous theorems.

The only significant difference between (i) and (ii) is at the fence immediately before the entry $2^{0}=1$. In (i) the pattern of the previous segments is followed at that fence, but in (ii) the missing difference $-3^{-1}=(n-1) / 3$ is compensated for at the fence $-2 \cdot 3^{-1} \mid 1$, i.e. $(2 n-2) / 3 \mid 1$, the fence difference being a reduced difference $n-2-((2 n-2) / 3)+1=(n-1) / 3$.

Note 3.7. In the range $3<n<1,000$, Theorem 3.7 covers only $n=31$. Failures arise as follows:

\begin{tabular}{c|cccc}
\hline$(n, h)$ & $(127,9)$ & $(151,5)$ & $(223,3)$ & $(631,7)$ \\
A reason for failure & $3^{-3}$ is even & $3^{-2}$ is even & $3^{-2}$ is even & $3^{-6}$ is even \\
\hline
\end{tabular}


EXAMPLES 3.7. With $(n, h)=(31,3)$, Theorem 3.7 yields terraces as follows: For type (i), we can take $c=5$ or 9 ; with $c=5$ we obtain the $\mathbb{Z}_{29}$ terrace

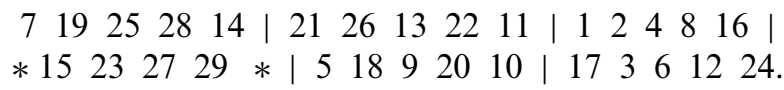

For type (ii), we can take only $c=11$, which yields the $\mathbb{Z}_{29}$ terrace

$$
\begin{aligned}
& \begin{array}{lllll|lllll|lllll|}
24 & 12 & 6 & 3 & 17 & 10 & 5 & 18 & 9 & 20 & 1 & 2 & 4 & 8 & 16
\end{array} \\
& \text { * } 15232729 * \mid \begin{array}{lllll|llll|}
11 & 21 & 26 & 13 & 22 & 25 & 19 & 74 & 14
\end{array} \text {. }
\end{aligned}
$$

THEOREM 3.8. Let $n$ be a prime, $n \equiv 17(\bmod 24)$, such that $\mathbb{Z}_{n} \backslash\{0\}=\langle 2,3\rangle$ and such that $\operatorname{ord}_{n}(2)$ is odd and equal to $(n-1) / 2 h$, where $h>1$. Suppose that the integers $-3^{-1},-3^{-2}, \ldots,-3^{-(h-1)}$ are all odd and that $-3^{1},-3^{2}, \ldots,-3^{+(h-1)}$ are all even. Then the following sequence, evaluated as described in $\S 1$, is a terrace for $\mathbb{Z}_{n-2}$ :

$$
\begin{aligned}
& \begin{array}{lllllllllll}
2^{0} & 2^{1} & \ldots & 2^{-1} & \mid * & -2^{-1} & -2^{-2} & \ldots & -2^{1} & * 1
\end{array} \\
& -3^{h-1} \cdot 2^{-1} \stackrel{2}{\leftarrow}\left|-3^{h-2} \cdot 2^{1} \stackrel{2}{\rightarrow}\right|-3^{h-3} \cdot 2^{1} \stackrel{2}{\rightarrow}|\cdots|-3^{1} \cdot 2^{1} \stackrel{2}{\rightarrow} \mid \\
& -3^{-1} \cdot 2^{1} \stackrel{2}{\rightarrow}\left|-3^{-2} \cdot 2^{1} \stackrel{2}{\rightarrow}\right| \cdots \mid-3^{-(h-1)} \cdot 2^{1} \stackrel{2}{\rightarrow} .
\end{aligned}
$$

Proof. The conditions on $n$ require 2 to be a square and 3 to be a non-square in $\mathbb{Z}_{n}$, so $n \equiv 7$ or $17(\bmod 24)$. We take $n \equiv 17(\bmod 24)$ to ensure that $-3^{-1} \cdot 2=$ $(n-2) / 3<n / 2$.

The proof follows the lines of previous proofs, special attention being required only by the fence $-3 \mid-3^{-1} \cdot 2$, where the difference must compensate for the missing difference $-3^{-1}$. If $n \equiv 17(\bmod 24)$, the entries at the fence are $n-3 \mid(n-2) / 3$, which give the reduced difference $(n+1) / 3$, the same as the missing difference $3^{-1}$. Contrariwise, if we were to try $n \equiv 7(\bmod 24)$, we would have $n-3 \mid(2 n-2) / 3$, which yields the difference $(n-7) / 3$, which does not match the missing difference $-3^{-1}=(n-1) / 3$.

Note 3.8. In the range $3<n<1,000$, Theorem 3.8 covers only $(n, h)=(233,4)$ and $(881,8)$.

EXAMPLE 3.8. With $(n, h)=(233,4)$, Theorem 3.8 yields the $\mathbb{Z}_{231}$ terrace

$$
\begin{aligned}
& \begin{array}{llll|llllll|llllllll}
1 & 2 & \ldots & 117 & * & 116 & 58 & \ldots & 231 & * & 103 & 168 & \ldots & 206 & 215 & 197 & \ldots & 224
\end{array} \\
& \begin{array}{llll|llll|lllllllll}
227 & 221 & \ldots & 230 & 77 & 154 & \ldots & 155 & 181 & 129 & \ldots & 207 & 138 & 43 & \ldots & 69
\end{array}
\end{aligned}
$$

4. Some terraces with segments of irregular lengths. We start this section of the paper with a construction for half-and-half terraces.

THEOREM 4.1. Let $n$ be a prime, $n>3$, that has 2 as a primitive root. Then the following sequences, evaluated as described in $\S 1$, are terraces for $\mathbb{Z}_{n-2}$ :

(i)

$$
!-3 \cdot 2^{0}-3 \cdot 2^{1} \ldots-2^{-1} ! \mid !-3 \cdot 2^{-1} \quad-3 \cdot 2^{-2} \ldots-2^{1} !
$$

as in (i) save that the segments are interchanged. 
For $n>5$, the terraces in (i) are half-and-half if and only if $3 \equiv 2^{i}(\bmod n)$, where $2<i<(n-1) / 2$, whilst those in (ii) are half-and-half if and only if $3 \equiv 2^{i}(\bmod n)$, where $(n+1) / 2<i<n-1$.

Proof.

(i) If $3=2^{\alpha}$, then the sequence is

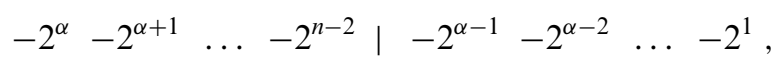

which includes all $-2^{i}$ except $-2^{0}=n-1$. The missing differences are $(n-1) / 2,(n-3) / 2$ and 1 , and the fence difference is 1 . As $(n-1) / 2 \equiv$ $-(n-3) / 2$ in $\mathbb{Z}_{n-2}$, the difference $(n-1) / 2$ is lost twice, as required.

If the second segment is the longer one, i.e. if $\alpha>n / 2$, it will give 1 as the $m$ th difference from the right, where $n=2 m+1$, i.e. the $(m-1)$ th difference from the left of the terrace. But this, with the 1 at the fence, will give 1 twice as a difference in the left half of the terrace, so that the terrace is not half-and-half. If $\alpha<n / 2$, the first $(n-1) / 2$ differences are all different, whence the terrace is half-and-half.

(ii) The proof is similar to that for (i). In any of the half-and-half terraces, the longer segment is on the left.

EXAMPLES 4.1. With $n=11$, Theorem 4.1 yields the following $\mathbb{Z}_{9}$ terrace, which is not half-and-half:

$$
\text { ! } 85 \text { !|!!4 } 2116379 \text { !. }
$$

With $n=13$, Theorem 4.1 yields the following half-and-half $\mathbb{Z}_{11}$ terrace:

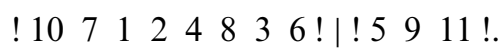

THEOREM 4.2. Let $n$ be a prime, $n \equiv 7(\bmod 8)$, such that $\operatorname{ord}_{n}(2)=(n-1) / 2$. Then the following sequences, evaluated as described in $\S 1$, are terraces for $\mathbb{Z}_{n-2}$ :

(i) If $+3 \in\langle 2\rangle$,

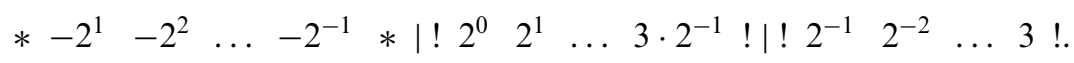

(ii) If $-3 \in\langle 2\rangle$,

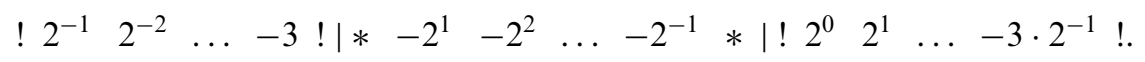

Proof. The condition $n \equiv 7(\bmod 8)$ ensures that 2 is a square in $\mathbb{Z}_{n}$ and that -1 is not. Thus $\langle 2\rangle$ is the set of squares, $-1 \notin\langle 2\rangle$, and $\mathbb{Z}_{n} \backslash\{0\}=\langle 2\rangle \cup-\langle 2\rangle$.

(i) Suppose that $3 \in\langle 2\rangle$. The missing differences are $(n-1) / 2,-(n+3) / 2=$ $(n-3) / 2,1$ and $(n-1) / 2$, whereas the fence differences are $(n-3) / 2$ and 1 . So we lose $(n-1) / 2$ twice, as required.

(ii) Suppose that $3 \notin\langle 2\rangle$. The missing differences are $(n-1) / 2$ (twice), 1 and $(n-3) / 2$, whereas the fence differences are 1 and $(n-3) / 2$. 
EXAMPLE 4.2. For $n=23$ we have $3 \in\langle 2\rangle$, so Theorem 4.2 yields the following $\mathbb{Z}_{21}$ terrace:

$$
\text { * } 211915714510201711 * \mid ! ! 1244816901813 \text { !|!!12 } 63 \text { !. }
$$

THeOREM 4.3. Let $n$ be a prime, $n \equiv 1(\bmod 24)$, such that $\operatorname{ord}_{n}(2)=(n-1) / 2$. Suppose that $c$ is an integer satisfying $0<c<(n-1) / 2$ and $c \notin\langle 2\rangle$. Then the following sequence, evaluated as described in $\S 1$, is a terrace for $\mathbb{Z}_{n-2}$ :

$$
2 c \stackrel{2}{\rightarrow}\left|!-2^{1}-2^{2} \ldots-3 \cdot 2^{-1} !\right| !-2^{-1}-2^{-2} \ldots-3 \quad \text { !. }
$$

Proof. The condition $n \equiv 1(\bmod 24)$ ensures that 2,3 and -1 are all squares in $\mathbb{Z}_{n}$. The proof is straightforward.

Note 4.3 . When $n \equiv 1(\bmod 4), x$ is a square in $\mathbb{Z}_{n}$ if and only if $n-x$ is a square. Accordingly, as $c$ in Theorem 4.3 is to be a non-square with $0<c<(n-1) / 2$, values of $c$ must exist for all values of $n$. In the range $3<n<1,000$, Theorem 4.3 covers $n$-values, with specimen $c$-values, as follows: $(n, c)=(97,7),(193,5),(313,7),(409,7)$ and $(769,7)$.

EXAMPLE 4.3. Taking $(n, c)=(97,7)$ in Theorem 4.3 yields the following $\mathbb{Z}_{95}$ terrace:

$$
\underbrace{1428 \ldots 527}_{48 \text { elements }}|! \underbrace{9593 \ldots 7247}_{18 \text { elements }} !| ! \underbrace{48 \quad 24 \ldots 9194}_{29 \text { elements }} \text { !. }
$$

THEOREM 4.4. Let $n$ be a prime, $n \equiv 1(\bmod 6)$, such that $\operatorname{ord}_{n}(2)=(n-1) / 3$ and $\mathbb{Z}_{n} \backslash\{0\}=\langle 2,3\rangle$. Suppose that $5 \in 3\langle 2\rangle$. Then the following sequence, evaluated as described in $\S 1$, is a terrace for $\mathbb{Z}_{n-2}$ :

$$
\begin{aligned}
& !-5 \cdot 2^{0}-5 \cdot 2^{1} \ldots-3 \cdot 2^{-1} \text { !|! } \quad-5 \cdot 2^{-1}-5 \cdot 2^{-2} \ldots-3 \text { !| }
\end{aligned}
$$

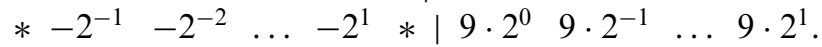

Proof. We have $\mathbb{Z}_{n} \backslash\{0\}=\langle 2\rangle \cup 3\langle 2\rangle \cup 9\langle 2\rangle$. As $\operatorname{ord}_{n}(2)$ is even we have $-1 \in\langle 2\rangle$, so that $-\langle 2\rangle=\langle 2\rangle$.

The missing differences are $(n-3) / 2,(n-5) / 2,(n-1) / 2,1$ and 9 . The fence differences are $1, n-3-((n-1) / 2)=(n-5) / 2$ and 9 (reduced). So we lose $(n-1) / 2$ and $(n-3) / 2$. But in $\mathbb{Z}_{n-2}$ we have $(n-3) / 2=-(n-1) / 2$.

Note 4.4. In the range $3<n<1,000$, Theorem 4.4 covers $n=43,109$ and 157.

EXAMPLE 4.4. Theorem 4.4 yields the $\mathbb{Z}_{41}$ terrace

$$
\begin{array}{rlllllllllll|llll}
! 38 & 33 & 23 & 3 & 6 & 12 & 24 & 5 & 10 & 20 ! \mid ! & 19 & 31 & 37 & 40 & \text { !| } \\
* & 21 & 32 & 16 & 8 & 4 & 2 & 1 & 22 & 11 & 27 & 35 & 39 & 41 & * & \text { | } \\
9 & 26 & 13 & 28 & 14 & 7 & 25 & 34 & 17 & 30 & 15 & 29 & 36 & 18 .
\end{array}
$$


5. Theorems for $n=p^{r}, r>1$. Our first theorem in this section is similar to Theorem 5.3 of $[6]$.

THEOREM 5.1. Let $n=3^{r}$, where $r>2$. Then the following sequence, evaluated as described in $\S 1$, is a terrace for $\mathbb{Z}_{n-2}$ :

$$
\begin{gathered}
: 12 \ldots(n-1) / 2:|:(n+1) / 2 \stackrel{2}{\leftarrow} n-2 *:| 3^{r-1} 2 \cdot 3^{r-1} \mid \\
4 \cdot 3^{r-2} \stackrel{2}{\rightarrow}\left|4 \cdot 3^{r-3} \stackrel{2}{\rightarrow}\right| \cdots \mid 4 \cdot 3 \stackrel{2}{\rightarrow} .
\end{gathered}
$$

Proof. The element 2 is a primitive root of $3^{i}$ for all $i \geq 1$. The missing differences are $(n-1) / 2$ (twice) $1,3^{r-1}, 2 \cdot 3^{r-2}, \ldots, 2 \cdot 3$. The fence differences are precisely these values apart from $(n-1) / 2$ (twice).

EXAMPLE 5.1. With $r=3$, Theorem 5.1 yields the following $\mathbb{Z}_{25}$ terrace:

$$
: \underbrace{12 \ldots 2013}_{9 \text { elements }}:|: \underbrace{147 \ldots 2325}_{8 \text { elements }} *:| 918 \mid 1224211536 .
$$

Note 5.1. If $r=2$, the $\mathbb{Z}_{7}$ terrace from Theorem 5.1 consists of the first three segments only:

$$
: 124:|: 57 *:| 36 .
$$

THEOREM 5.2. Let $n=p^{2}$, where $p$ is a prime, $p>3$, such that 2 is a primitive root of both $p$ and $n$. Write $s=(n-p) / 2=p(p-1) / 2$, and let $c$ be an integer satisfying $0<c<p / 2$. Then the following sequences, evaluated as described in $\S 1$, are terraces for $\mathbb{Z}_{n-2}$ :

(i)

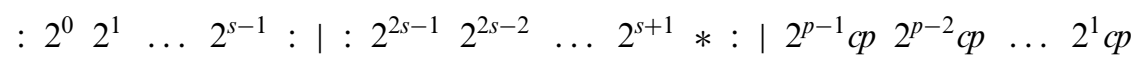

(ii)

$$
!-3 \cdot 2^{0}-3 \cdot 2^{1} \ldots-2^{-1} !\left|!-3 \cdot 2^{-1}-3 \cdot 2^{-2} \ldots-2^{1} !\right| 2^{p-1} c p 2^{p-2} c p \ldots 2^{1} c p
$$

Proof. For (i) we have $2^{s} \equiv-1(\bmod n)$. The missing differences are $(n-1) / 2$ (twice), 1 and $c p$. The last two of these are compensated for by the fence differences. for $\mathbb{Z}_{23}$ :

EXAMPLE 5.2. With $(n, p, c)=(25,5,1)$, Theorem 5.2 yields the following terraces

(i)

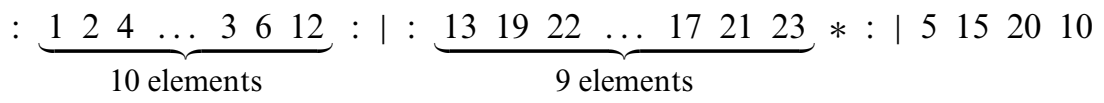

(ii)

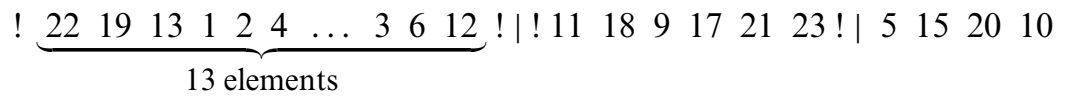


THEOREM 5.3. Let $n=p^{2}$, where $p$ is a prime, $p \equiv 7(\bmod 24)$, such that $\operatorname{ord}_{p}(2)=$ $(p-1) / 2$ and $\operatorname{ord}_{n}(2)=(n-p) / 2$. Write $s=(n-p) / 2$, and let $c$ be any integer satisfying both $0<c<p / 2$ and $c \neq \equiv 1(\bmod 3)$. Then the following sequence, evaluated as described in $\S 1$, is a terrace for $\mathbb{Z}_{n-2}$ :

$$
\begin{aligned}
& \begin{array}{llllllllllll}
2^{0} & 2^{1} & \ldots & 2^{s-1} & * & * & -2^{s-1} & -2^{s-2} & \ldots & -2^{1} & * & \text { । }
\end{array}
\end{aligned}
$$

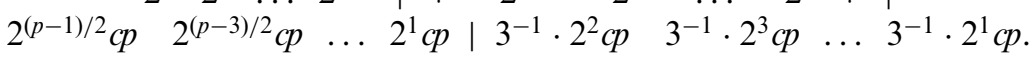

Proof. As $p \equiv 7(\bmod 12)$, the element 3 is not a square in $\mathbb{Z}_{p}$. As $p \equiv 7(\bmod 8)$, the element 2 is a square whereas -1 is not. Thus $\mathbb{Z}_{p} \backslash\{0\}=\langle 2\rangle \cup 3^{-1}\langle 2\rangle$, and $\langle 2\rangle \cup-\langle 2\rangle$ is the set of units in $\mathbb{Z}_{n}$.

Consider $3^{-1} \cdot 2 c$. If $c \equiv 0(\bmod 3)$, we have $3^{-1} \cdot 2 c=2 c / 3$; if $c \equiv 1(\bmod 3)$, it is $(p+2 c) / 3$, and if $c \equiv 2(\bmod 3)$ it is $(2 p+2 c) / 3$. For the construction to work, the missing difference at the end of the final segment has to be compensated for by the fence difference at the start of that segment. If $c \equiv 0(\bmod 3)$, both differences are $2 c p / 3$, which is less than $n / 2$, and if $c \equiv 2(\bmod 3)$ they are both $p(p-2 c) / 3$. However, when $c \equiv 1(\bmod 3)$, the missing difference is $p(p+2 c) / 3$ if $c<p / 4$ and $2 p(p-c) / 3$ if $c>$ $p / 4$, whereas the fence difference is $n-2-\{2 p(p+2 c) / 3-2 p c\}=\{p(p+2 c) / 3\}-2$ if $c<p / 4$ and is similarly $\{2 p(p-c) / 3\}-2$ if $c>p / 4$.

EXAmple 5.3. For $(n, p, c)=(49,7,2)$, Theorem 5.3 yields the following terrace for $\mathbb{Z}_{47}$ :

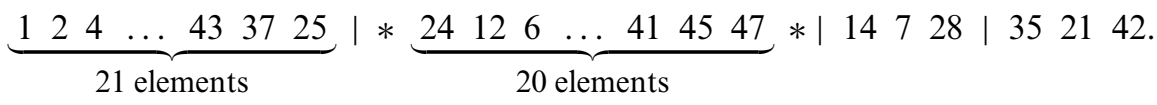

THEOREM 5.4. Let $n=p^{2}$, where $p$ is a prime, $p \equiv 17(\bmod 24)$, such that $\operatorname{ord}_{p}(2)=$ $(p-1) / 2$ and $\operatorname{ord}_{n}(2)=(n-p) / 2$. Write $s=(n-p) / 2$, and let c be any integer satisfying $0<c<p / 6$. Then the following sequence, evaluated as described in $\S 1$, is a terrace for $\mathbb{Z}_{n-2}$ :

$$
\begin{array}{cccccccccc}
-3 \cdot 2^{0}-3 \cdot 2^{1} & \ldots & -3 \cdot 2^{s-1} & * & -2^{s-1} & -2^{s-2} & \ldots & -2^{1} * \mid \\
3 \cdot 2^{(p-1) / 2} c p & 3 \cdot 2^{(p-3) / 2} c p & \ldots & 3 \cdot 2^{1} c p & 2^{2} c p & 2^{3} c p & \ldots & 2^{1} c p .
\end{array}
$$

Proof. As $p \equiv 5(\bmod 12)$, the element 3 is not a square in $\mathbb{Z}_{p}$ or $\mathbb{Z}_{n}$. As $p \equiv 1$ (mod 4$)$, the element -1 is a square and therefore lies in $\langle 2\rangle$. Thus $-\langle 2\rangle \cup-3\langle 2\rangle$ is the set of units (in $\mathbb{Z}_{p}$ or $\mathbb{Z}_{n}$ ).

The missing differences are $(n-3) / 2,(n-1) / 2,1,3 c p$ and $2 c p$. The fence differences compensate for the last three of these. The result follows as, in $\mathbb{Z}_{n-2}$, we have $(n-3) / 2=-(n-1) / 2$.

EXAMPLE 5.4. With $(n, p, c)=(289,17,1)$, Theorem 5.4 yields the following terrace for $\mathbb{Z}_{287}$ :

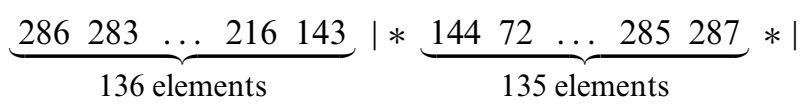

\begin{tabular}{llllllll|llllllll}
51 & 170 & 85 & 187 & 238 & 119 & 204 & 102 & 68 & 136 & 272 & 255 & 221 & 153 & 17 & 34.
\end{tabular} 
6. Ad hoc constructions. By judicious use of reduced differences, we have been able to construct $\mathbb{Z}_{n-2}$ terraces, via $\mathbb{Z}_{n}$, for $n=73,151,331$ and 641 , thereby filling some of the gaps left by the succession of constructions in preceding sections of this paper. We now present these terraces in forms so similar to those used heretofore that the reader should need no further explanations of them save that in each terrace, a reduced difference occurs at the fence immediately following the element $d$ :

$n=73$, with $c=7, d=54$ :

$$
\begin{gathered}
-3^{-1} \stackrel{2}{\leftarrow}-2 \cdot 3^{-1}\left|1 \stackrel{2}{\rightarrow} 2^{-1}\right| *(n-1) / 2 \stackrel{2}{\leftarrow} n-2 * \mid \\
3^{3} \cdot 2 c \stackrel{2}{\rightarrow} 3^{3} c\left|3^{2} \cdot 2 c \stackrel{2}{\rightarrow} 3^{2} c\right| 3^{1} \cdot 2 c \stackrel{2}{\leftarrow} d \mid
\end{gathered}
$$

$n=151$, with $c=103, d=40$ :

$$
\begin{gathered}
3 \stackrel{2}{\rightarrow} 2^{-1} \cdot 3\left|2^{-1} \stackrel{2}{\leftarrow} 1\right| *(n-1) / 2 \stackrel{2}{\leftarrow} n-2 * \mid \\
3^{2} \cdot 2^{-1} d \stackrel{2}{\leftarrow} 3^{2} d\left|3^{1} \cdot 2 d \stackrel{2}{\rightarrow} 3^{1} d\right| 3^{0} \cdot 2 d \stackrel{2}{\rightarrow} 3^{0} d \mid \\
3^{3} \cdot 2 c \stackrel{2}{\rightarrow} 3^{3} c\left|3^{2} \cdot 2 c \stackrel{2}{\rightarrow} 3^{2} c\right| 3^{1} \cdot 2 c \stackrel{2}{\rightarrow} 3^{1} c \mid 3^{0} \cdot 2 c \stackrel{2}{\rightarrow} 3^{0} c .
\end{gathered}
$$

$n=331$, with $c=3, d=270$ :

$$
\begin{gathered}
+3^{-1} \stackrel{2}{\leftarrow}+2 \cdot 3^{-1}\left|: 1 \stackrel{2}{\rightarrow}-2^{-1}:\right|:+2^{-1} \stackrel{2}{\leftarrow}-2^{1} *: \mid \\
3^{1} \cdot 2^{-1} d \stackrel{2}{\leftarrow} 3^{1} d\left|3^{0} \cdot 2 d \stackrel{2}{\rightarrow} 3^{0} d\right| \\
3^{6} \cdot 2 c \stackrel{2}{\rightarrow} 3^{6} c\left|3^{5} \cdot 2 c \stackrel{2}{\rightarrow} 3^{5} c\right| \cdots \mid 3^{0} \cdot 2 c \stackrel{2}{\rightarrow} 3^{0} c .
\end{gathered}
$$

$n=641$, with $c=635, d=374$ :

$$
\begin{gathered}
: 1 \stackrel{2}{\rightarrow}-2^{-1}:\left|:+2^{-1} \stackrel{2}{\leftarrow}-2^{1} *:\right| \\
2^{-1} d \stackrel{2}{\leftarrow} d \mid \\
3^{7} \cdot 2 c \stackrel{2}{\rightarrow} 3^{7} c\left|3^{6} \cdot 2 c \stackrel{2}{\rightarrow} 3^{6} c\right| \cdots \mid 3^{0} \cdot 2 c \stackrel{2}{\rightarrow} 3^{0} c .
\end{gathered}
$$

7. Table. We now give a table showing which theorems or section of the paper cover each of the primes $n$ in the range $3<n<1,000$. Theorems producing narcissistic terraces for $\mathbb{Z}_{n-2}$ are marked with an asterisk.

\begin{tabular}{rl}
$n$ & Theorems \\
\hline 5 & $2.1^{*}, 4.1$ \\
7 & $2.2^{*}, 4.2$ \\
11 & $2.1^{*}, 4.1$ \\
13 & $2.1^{*}, 4.1$ \\
17 & $2.3,2.4,3.1^{*}$ \\
19 & $2.1^{*}, 4.1$ \\
23 & $2.2^{*}, 4.2$ \\
29 & $2.1^{*}, 4.1$ \\
31 & $2.8,3.7$ \\
37 & $2.1^{*} .4 .1$ \\
41 & $2.3,2.4,3.1^{*}$ \\
\hline
\end{tabular}

\begin{tabular}{rl}
$n$ & Theorems \\
\hline 43 & $2.5,2.6,3.1^{*}, 3.2,3.4,4.4$ \\
47 & $2.2^{*}, 4.2$ \\
53 & $2.1^{*}, 4.1$ \\
59 & $2.1^{*}, 4.1$ \\
61 & $2.1^{*}, 4.1$ \\
67 & $2.1^{*}, 4.1$ \\
71 & $2.2^{*}, 4.2$ \\
73 & $\S 6$ \\
79 & $2.2^{*}, 4.2$ \\
83 & $2.1^{*}, 4.1$ \\
89 & $3.6^{*}$ \\
\hline
\end{tabular}




\begin{tabular}{|c|c|c|c|}
\hline$n$ & Theorems & $n$ & Theorems \\
\hline 97 & $2.3,4.3$ & 367 & $2.2^{*}, 4.2$ \\
\hline 101 & $2.1^{*}, 4.1$ & 373 & $2.1^{*}, 4.1$ \\
\hline 103 & $2.2^{*}, 4.2$ & 379 & $2.1^{*}, 4.1$ \\
\hline 107 & $2.1^{*}, 4.1$ & 383 & $2.2^{*}, 4.2$ \\
\hline 109 & $2.5,2.6,3.1^{*} 3.2,3.3,3.4,4.4$ & 389 & $2.1^{*}, 4.1$ \\
\hline 113 & $2.7,3.2$ & 397 & $3.2,3.4$ \\
\hline 127 & - & 401 & $2.3,2.4,3.1^{*}$ \\
\hline 131 & $2.1^{*}, 4.1$ & 409 & $2.3,4.3$ \\
\hline 137 & $2.3,2.4,3.1^{*}$ & 419 & $2.1^{*}, 4.1$ \\
\hline 139 & $2.1^{*}, 4.1$ & 421 & $2.1^{*}, 4.1$ \\
\hline 149 & $2.1^{*}, 4.1$ & 431 & - \\
\hline 151 & $\S 6$ & 433 & $2.10,3.5$ \\
\hline 157 & $2.5,2.6,3.2,3.3,3.4,4.4$ & 439 & 2.9 \\
\hline 163 & $2.1^{*}, 4.1$ & 443 & $2.1^{*}, 4.1$ \\
\hline 167 & $2.2^{*}, 4.2$ & 449 & $2.3,2.4,3.1^{*}$ \\
\hline 173 & $2.1^{*}, 4.1$ & 457 & 2.10 \\
\hline 179 & $2.1^{*}, 4.1$ & 461 & $2.1^{*}, 4.1$ \\
\hline 181 & $2.1^{*}, 4.1$ & 463 & $2.2^{*}, 4.2$ \\
\hline 191 & $2.2,4.2$ & 467 & $2.1^{*}, 4.1$ \\
\hline 193 & $2.3,4.3$ & 479 & $2.2^{*}, 4.2$ \\
\hline 197 & $2.1^{*}, 4.1$ & 487 & $2.2^{*}, 4.2$ \\
\hline 199 & $2.2^{*}, 4.2$ & 491 & $2.1^{*}, 4.1$ \\
\hline 211 & $2.1^{*}, 4.1$ & 499 & 2.6 \\
\hline 223 & $2.8,3.6^{*}$ & 503 & $2.2^{*}, 4.2$ \\
\hline 227 & $2.1^{*}, 4.1$ & 509 & $2.1^{*}, 4.1$ \\
\hline 229 & $2.5,2.6,3.2,3.3,3.4$ & 521 & $2.3,2.4,3.1^{*}$ \\
\hline 233 & 3.8 & 523 & $2.1^{*}, 4.1$ \\
\hline 239 & $2.2^{*}, 4.2$ & 541 & $2.1^{*}, 4.1$ \\
\hline 241 & - & 547 & $2.1^{*}, 4.1$ \\
\hline 251 & 3.2 & 557 & $2.1^{*}, 4.1$ \\
\hline 257 & - & 563 & $2.1^{*}, 4.1$ \\
\hline 263 & $2.2^{*}, 4.2$ & 569 & $2.3,2.4,3.1^{*}$ \\
\hline 269 & $2.1^{*}, 4.1$ & 571 & $3.2,3.3,3.4$ \\
\hline 271 & $2.2^{*}, 4.2$ & 577 & 2.7 \\
\hline 277 & $2.5,2.6,3.1^{*}, 3.2,3.4$ & 587 & $2.1^{*}, 4.1$ \\
\hline 281 & $2.7,3.1^{*}, 3.2$ & 593 & $2.7,3.1^{*}, 3.2$ \\
\hline 283 & $2.5,2.6,3.2,3.3,3.4$ & 599 & $2.2^{*}, 4.2$ \\
\hline 293 & $2.1^{*}, 4.1$ & 601 & - \\
\hline 307 & 2.6 & 607 & $2.2^{*}, 4.2$ \\
\hline 311 & $2.2^{*}, 4.2$ & 613 & $2.1^{*}, 4.1$ \\
\hline 313 & $2.3,4.3$ & 617 & $2.7,3.2$ \\
\hline 317 & $2.1^{*}, 4.1$ & 619 & $2.1^{*}, 4.1$ \\
\hline 331 & $\S 6$ & 631 & - \\
\hline 337 & - & 641 & $\S 6$ \\
\hline 347 & $2.1^{*}, 4.1$ & 643 & 2.6 \\
\hline 349 & $2.1^{*}, 4.1$ & 647 & $2.2^{*}, 4.2$ \\
\hline 353 & $2.7,3.1^{*}, 3.2$ & 653 & $2.1^{*}, 4.1$ \\
\hline 359 & $2.2^{*}, 4.2$ & 659 & $2.1^{*}, 4.1$ \\
\hline
\end{tabular}




\begin{tabular}{rl}
$n$ & Theorems \\
\hline 661 & $2.1^{*}, 4.1$ \\
673 & - \\
677 & $2.1^{*}, 4.1$ \\
683 & - \\
691 & $2.5,2.6,3.1^{*}, 3.2,3.4$ \\
701 & $2.1^{*}, 4.1$ \\
709 & $2.1^{*}, 4.1$ \\
719 & $2.2^{*}, 4.2$ \\
727 & 2.9 \\
733 & $2.5,2.6,3.2,3.3,3.4$ \\
739 & $2.5,2.6,3.1^{*}, 3.2,3.3,3.4$ \\
743 & $2.2^{*}, 4.2$ \\
751 & $2.2^{*}, 4.2$ \\
757 & $2.1^{*}, 4.1$ \\
761 & $2.3,2.4,3.1^{*}$ \\
769 & $2.3,4.3$ \\
773 & $2.1^{*}, 4.1$ \\
787 & $2.1^{*}, 4.1$ \\
797 & $2.1^{*}, 4.1$ \\
809 & $2.3,2.4,3.1^{*}$ \\
811 & $2.5,2.6,3.1^{*}, 3.2,3.3,3.4$ \\
821 & $2.1^{*}, 4.1$ \\
823 & $2.2^{*}, 4.2$ \\
827 & $2.1^{*}, 4.1$ \\
\hline & \\
&
\end{tabular}

\begin{tabular}{rl}
$n$ & Theorems \\
\hline 829 & $2.1^{*}, 4.1$ \\
839 & $2.2^{*}, 4.2$ \\
853 & $2.1^{*}, 4.1$ \\
857 & $2.3,2.4,3.1^{*}$ \\
859 & $2.1^{*}, 4.1$ \\
863 & $2.2^{*}, 4.2$ \\
877 & $2.1^{*}, 4.1$ \\
881 & $3.8^{*}$ \\
883 & $2.1^{*}, 4.1$ \\
887 & $2.2^{*}, 4.2$ \\
907 & $2.1^{*}, 4.1$ \\
911 & - \\
919 & 2.9 \\
929 & $2.3,2.4,3.1^{*}$ \\
937 & - \\
941 & $2.1^{*}, 4.1$ \\
947 & $2.1^{*}, 4.1$ \\
953 & - \\
967 & $2.2^{*}, 4.2$ \\
971 & $3.1^{*}, 3.2$ \\
977 & $2.3^{*}, 2.4,3.1^{*}$ \\
983 & $2.2^{*}, 4.2$ \\
991 & $2.2^{*}, 4.2$ \\
997 & 2.6 \\
\hline &
\end{tabular}

\section{REFERENCES}

1. I. Anderson and D. A. Preece, Locally balanced change-over designs, Utilitas Math. 62 (2002), 33-59.

2. I. Anderson and D. A. Preece, Power-sequence terraces for $\mathbb{Z}_{n}$ where $n$ is an odd prime power, Discrete Math. 261 (2003), 31-58.

3. I. Anderson and D. A. Preece, Some narcissistic half-and-half power-sequence $\mathbb{Z}_{n}$ terraces with segments of different lengths, Cong. Numer. 163 (2003), 5-26.

4. I. Anderson and D. A. Preece, Narcissistic half-and-half power-sequence terraces for $\mathbb{Z}_{n}$ with $n=p q^{t}$, Discrete Math. 279 (2004), 33-60.

5. I. Anderson and D. A. Preece, Some power-sequence terraces for $\mathbb{Z}_{p q}$ with as few segments as possible, Discrete Math. 293 (2005), 29-59.

6. I. Anderson and D. A. Preece, Some $\mathbb{Z}_{n-1}$ terraces from $\mathbb{Z}_{n}$ power-sequences, $n$ being an odd prime power, Proc. Edinbr. Math. Soc. 50 (2007), 527-549.

7. I. Anderson and D. A. Preece, Some $\mathbb{Z}_{n+2}$ terraces from $\mathbb{Z}_{n}$ power-sequences, $n$ being an odd prime, Discrete Math. 308 (2008), 4086- 4107.

8. R. A. Bailey, Quasi-complete Latin squares: Construction and randomisation, $J . R$. Statist. Soc. B 46 (1984), 323-334.

9. A. J. W. Hilton, M. Mays, C. A. Rodger and C. StJ. A. Nash-Williams, Hamiltonian double Latin squares, J. Combin. Theory B 87 (2003), 81-129.

10. G. Ringel, Map color theorem (Springer, Berlin, 1974). 